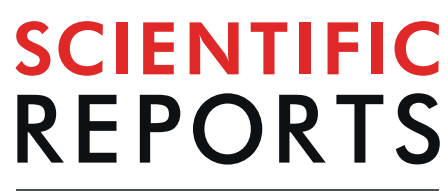

natureresearch

\title{
OPEN Semaphorin 6A Attenuates the Migration Capability of Lung Cancer Cells via the NRF2/HMOX1 Axis
}

Received: 2 May 2018

Accepted: 30 August 2019

Published online: 16 September 2019
Li-Han Chen ${ }^{1}$, Che-Yu Liao ${ }^{2}$, Liang-Chuan Lai ${ }^{3,4}{ }^{3}$, Mong-Hsun Tsai ${ }^{2,4,5,6,7}$ \& EricY. Chuang ${ }^{1,6,8,9}$

Cell migration is a fundamental feature of cancer recurrence. Since recurrence is correlated with high mortality in lung cancer, it follows that reducing cell migration would decrease recurrence and increase survival rates. Semaphorin-6A (SEMA6A), a protein initially known as a regulator of axonal guidance, is down-regulated in lung cancer tissue, and low levels of SEMA6A are associated with cancer recurrence. Thus, we hypothesized that SEMA6A could suppress cancer cell migration. In this study, we found that the migration capability of $\mathrm{H} 1299$ lung cancer cells decreased with SEMA6A overexpression, while it increased with SEMA6A silencing. Moreover, silencing of the cellular homeostasis protein Heme-oxygenase-1 (HMOX1) and/or the transcription factor Nuclear Factor, Erythroid-2-Like-2 (NRF2) reversed the migration-suppressing effect of SEMA6A and the SEMA6A-driven alterations in expression of urokinase insulin-like-growth-factor-binding-protein-3, Matrix metalloproteinase (MMP)-1, and MMP9, the downstream effectors of HMOX1. Taken together, these results demonstrate that SEMA6A is a potential suppressor of cancer migration that functions through the NRF2/HMOX1 axis. Our results explain why low SEMA6A is linked to high recurrence in the clinical setting and suggest that SEMA6A could be useful as a biomarker or target in lung cancer therapy.

Increases in cell migration, invasion, and metastasis are hallmarks of malignancy ${ }^{1}$. Metastasis has also been reported as a dominant cause of recurrence and a major obstacle to success in the treatment of cancer ${ }^{2,3}$. In the United States, lung cancer patients' 5-year survival rate is lower in the regional stage (28\%) than in the localized stage (55\%). Moreover, the 5 -year survival rate is only $4 \%$ in distant-stage patients ${ }^{4}$. Since mortality steeply increases when metastasis occurs in lung cancer patients, investigation of the genes involved in suppression of metastasis should make a significant contribution to lung cancer therapy.

Semaphorins were initially reported to regulate short-range axonal guidance. More recently, the role of semaphorins in cell migration, carcinogenesis, and cancer metastasis has been addressed in several studies. For example, semaphorins $3 \mathrm{~A}, 3 \mathrm{~B}$, and $3 \mathrm{~F}$ reduced growth and metastasis in melanoma, breast cancer, and colorectal cancer, respectively ${ }^{5-7}$. Similar to these tumor suppressor semaphorins, semaphorin 6A (SEMA6A) significantly inhibited the growth of lung cancer cells when losing either SEMA domain or whole extracellular region ${ }^{8}$. Moreover, we observed that SEMA6A was down-regulated in lung cancer tissue compared to its adjacent normal tissue $^{9}$. However, the role of SEMA6A in cancer progression has been examined in only a few studies ${ }^{9,10}$.

SEMA6A is a single-pass transmembrane protein ${ }^{11-14}$ and can act as either a ligand or a receptor ${ }^{15-18}$. The extracellular region of SEMA6A controls the guidance of axons in embryonic development, and the intracellular region has been revealed to guide the navigation of axons by reorganizing the neuronal cytoskeleton ${ }^{18}$. Since both axonal guidance and metastasis are associated with the alteration of cell morphology and cell movement, SEMA6A may also regulate the migration of lung cancer cells. Although Chien et al. (2017) linked low

\footnotetext{
${ }^{1}$ Graduate Institute of Biomedical Electronics and Bioinformatics, National Taiwan University, Taipei, Taiwan. ${ }^{2}$ Institute of Biotechnology, National Taiwan University, Taipei, Taiwan. ${ }^{3}$ Institute of Physiology, National Taiwan University, Taipei, Taiwan. ${ }^{4}$ Genome and Systems Biology Degree Program, National Taiwan University, Taipei, Taiwan. ${ }^{5}$ Center for Biotechnology, National Taiwan University, Taipei, Taiwan. ${ }^{6}$ Bioinformatics and Biostatistics Core, Center of Genomic Medicine, National Taiwan University, Taipei, Taiwan. ${ }^{7}$ Agricultural Biotechnology Research Center, Academia Sinica, Taipei, Taiwan. ${ }^{8}$ School of Chinese Medicine, China Medical University, Taichung, Taiwan. ${ }^{9}$ Biomedical Technology and Device Research Laboratories, Industrial Technology Research Institute, Hsinchu, Taiwan. Correspondence and requests for materials should be addressed to M.-H.T. (email: motiont@gmail.com) or E.Y.C. (email: chuangey@ntu.edu.tw)
} 
A

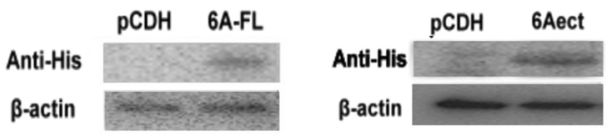

C

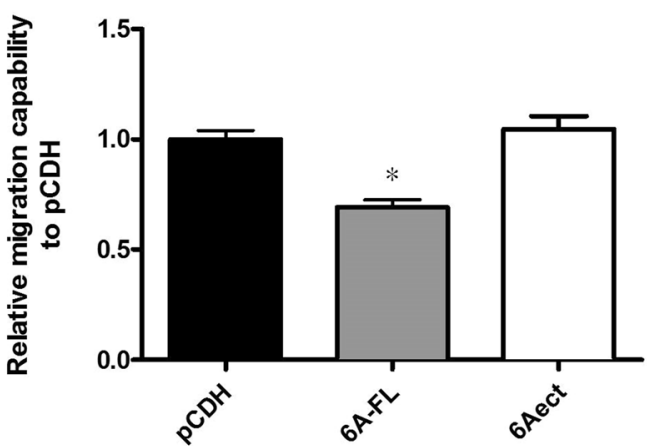

B

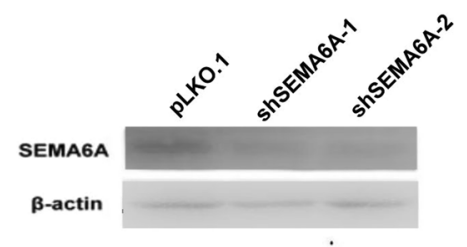

D

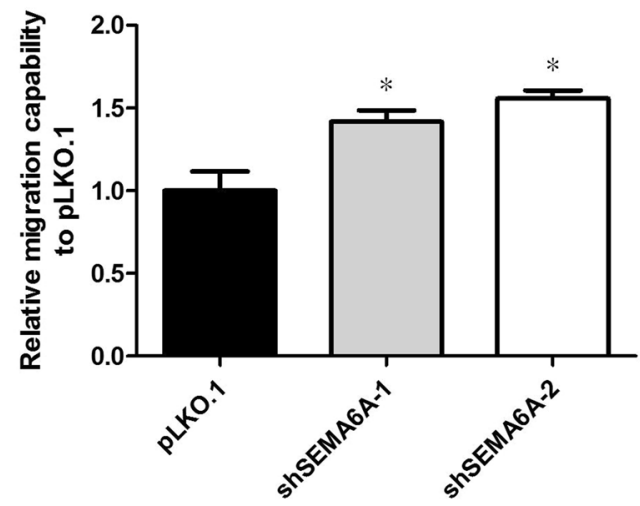

Figure 1. Effect of SEMA6A expression in H1299 cells on migration by transwell assay. (A) Western blot of His-tagged 6A-FL and 6Aect. (B) Western blot of SEMA6A. (C) Migration capability of 6A-FL- and 6Aectoverexpressing H1299 cells. (D) Migration capability of H1299 cells with SEMA6A silenced by two different shRNAs. *Statistical significance compared to empty vector-transfected H1299 cells at $p<0.05, \mathrm{n}=3$.

expression of SEMA6A to recurrence by analyzing the data from 504 papillary thyroid cancer patients ${ }^{19}$, the effect of SEMA6A on migration in cancer cells has not been investigated.

Based on our current knowledge of SEMA6A, we hypothesized that SEMA6A can reduce the migration of lung cancer cells. To test this hypothesis, we compared the migration capability of H1299 lung cancer cells with and without SEMA6A overexpression. Microarray data from H1299 cells overexpressing SEMA6A versus control cells were also analyzed to identify genes potentially involved in the SEMA6A-related signaling pathway of migration. Finally, gene regulation techniques were used to confirm the effects of this pathway.

\section{Results}

SEMA6A's regulation of $\mathrm{H} 1299$ cell migration requires its cytosolic region. For investigating the effect of SEMA6A on lung cancer cell migration, we overexpressed either the full-length DNA constructs of SEMA6A (6A-FL) or the DNA construct of the ectodomain (6Aect, residues 1-704) (Fig. 1A), silenced endogenous SEMA6A (Fig. 1B) in H1299 cells, and performed a transwell assay. The migration capability of 6A-FL-overexpressing cells decreased by approximately $35 \%$ compared to the empty vector-transfected control cells (Fig. 1C). On the other hand, SEMA6A-silenced H1299 cells had a higher migration rate than empty vector-transfected cells (Fig. 1D). Moreover, there was no difference between 6Aect-overexpressing cells and control cells (Fig. 1C). Since the 6Aect construct, composed of only the transmembrane region and extracellular ectodomain of SEMA6A, could not decrease cell migration, the cytosolic region of SEMA6A must be necessary for the migration-reducing effect.

Heme oxygenase 1 (HMOX1) is induced in SEMA6A-overexpressing H1299 cells. After SEMA6A was demonstrated to modulate lung cancer cell migration, we wanted to identify the genes downstream of SEMA6A in the migration inhibition pathway. Microarray data from 6A-FL-overexpressing H1299 cells showed that the expression levels of 437 genes were 1.5 times greater compared to control cells (Fig. 2A). The 25 genes related to cell migration were selected by Ingenuity Pathway Analysis (IPA), and HMOX1 had the highest fold change among them: $18.1 \times$ with a $p$ value $<0.0026$ (Fig. $2 B$ ).

HMOX1 is the downstream regulator of SEMA6A in migration signaling in $\mathrm{H} 1299$ cells. Since SEMA6A overexpression induced HMOX1 in H1299 cells, we hypothesized that SEMA6A could regulate HMOX1 to attenuate cell migration. To explore the effect of HMOX1 on migration, we evaluated the migration capability of HMOX1-overexpressing and -silenced H1299 cells using the transwell assay. The mRNA levels of HMOX1 were successfully regulated in the H1299 cells (Fig. 3A). The results of this assay showed that the migration rates were lower in HMOX1-overexpressing cells (Fig. 3B) and higher in HMOX1-silenced cells (Fig. 3C) compared to the respective control cells. Thus, HMOX1 plays a role in inhibition of migration in H1299 cells. 
A

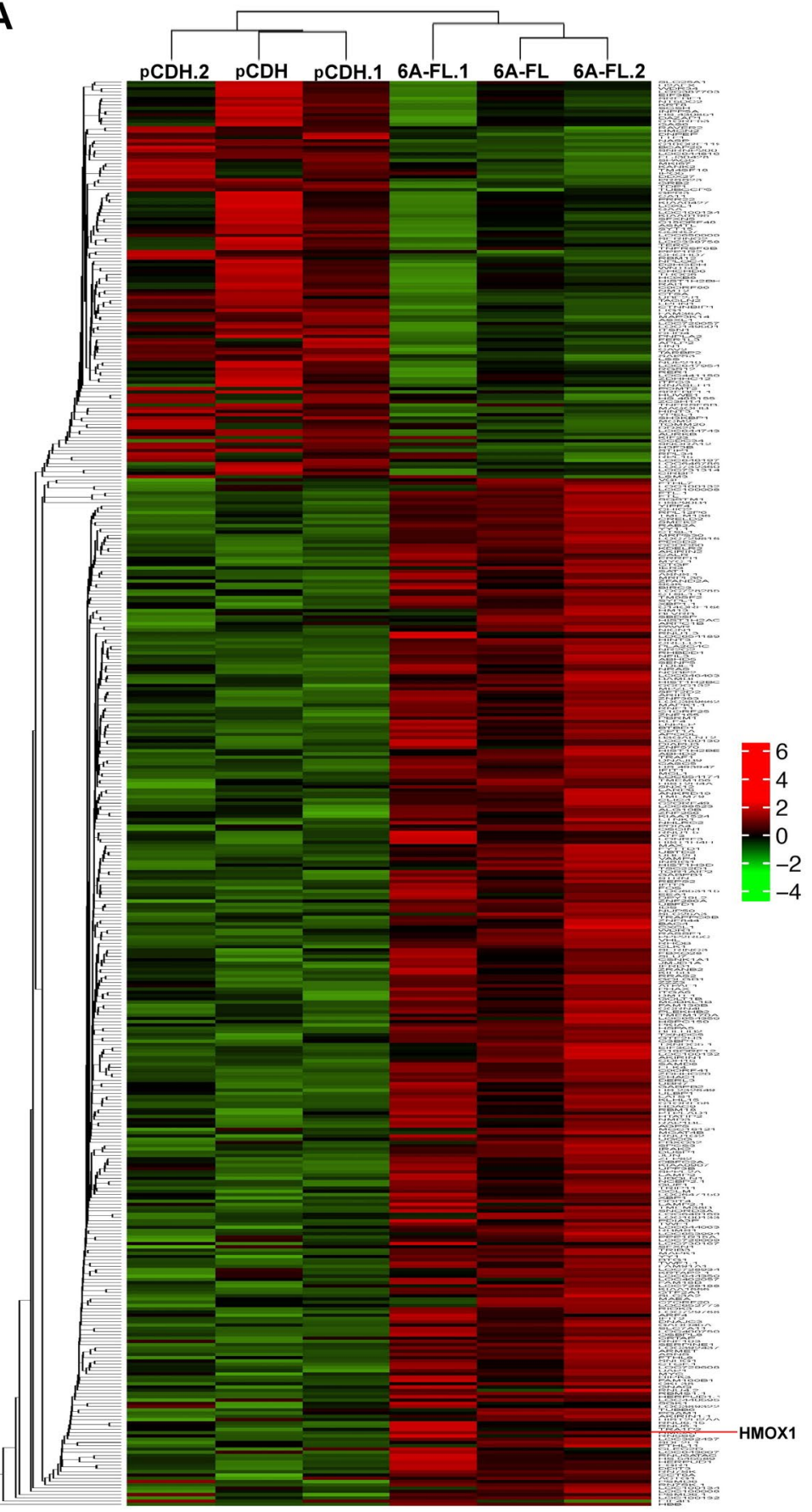

B

\begin{tabular}{lcc}
\hline Gene Symbols & Fold Change & p value \\
HMOX1 & 18.1 & 0.002501 \\
MAPK1 & 5.9 & 0.01007 \\
JUN & 4.6 & 0.002065 \\
SQSTM1 & 4.6 & 0.005313 \\
SERPINE1 & 4.2 & 0.006871 \\
ASNS & 4.0 & 0.002405 \\
HSPA5 & 3.7 & 0.001554 \\
VHL & 3.6 & 0.004668 \\
RHOB & 3.1 & 0.004045 \\
YY1 & 3.1 & 0.008052 \\
HTATIP2 & 2.9 & 0.008769 \\
WWTR1 & 2.7 & 0.002869 \\
CDKN1A & 2.6 & 0.002198 \\
HEY1 & 2.5 & 0.001411 \\
LRIG1 & 2.4 & 0.009706 \\
WARS & 2.4 & 0.002501 \\
LOX & 2.3 & 0.003239 \\
L3MBTL1 & 2.3 & 0.001335 \\
C3 & 2.3 & 0.00276 \\
MYOC & 1.8 & 0.002268 \\
CNTROB & 1.8 & 0.00698 \\
TWIST1 & 1.6 & 0.005625 \\
ARHGDIB & 1.6 & 0.004224 \\
MYH10 & 1.6 & 0.009713 \\
TAGLN2 & 1.5 & 0.005809 \\
\hline
\end{tabular}

Figure 2. Heatmap and hierarchical clustering of microarray profiles from the $\mathrm{pCDH}$-transfected (pCDH, pCDH.1, pCDH.2) and 6A-FL-overexpressing (6A-FL, 6A-FL.1, 6A-FL.2) H1299 cells (A), and the migrationrelated genes fold change $>1.5$ in 6A-FL-overexpressing H1299 cells (B).

Next, the involvement of HMOX1 in the action of SEMA6A in the H1299 cells was investigated by determining the migration capability of $\mathrm{H} 1299$ cells co-transfected with empty pLKO.1 and empty pCDH, empty pLKO.1 and 6A-FL, shHMOX1 and empty pCDH, or shHMOX1 and 6A-FL using transwell and wound-healing assays. The pLKO.1 + 6A-FL co-transfected H1299 cells had the highest expression levels of HMOX1, followed by the negative control H1299 cells (Fig. 3D,E). The levels of HMOX1 were the lowest in the shHMOX1 + pCDH cells and shHMOX1 + 6A-FL cells (Fig. 3D,E). The H1299 cells co-transfected with empty pLKO.1 and 6A-FL had a lower migration rate than the $\mathrm{H} 1299$ cells co-transfected with empty pLKO.1 and empty pCDH or with shHMOX1 and 6A-FL in transwell assay (Fig. 3F). Similarly, the cell-free area was larger in the empty pLKO.1 +6A-FL cells than in the other co-transfected cells in the wound-healing assay (Fig. 4). These results suggest that silencing HMOX1 reverses the SEMA6A-derived attenuation of migration in $\mathrm{H} 1299$ cells. 
A
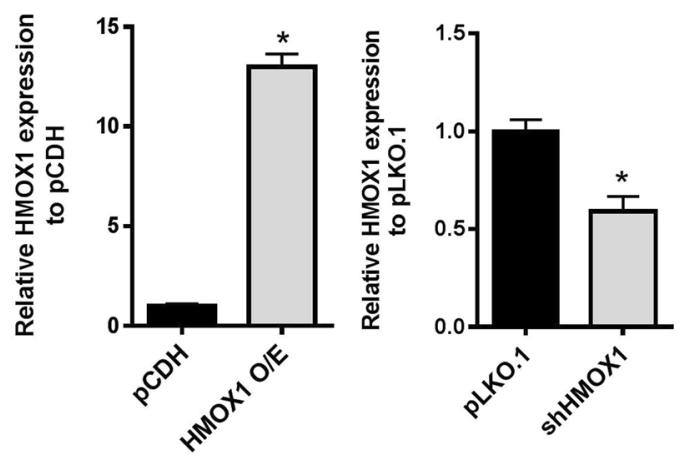

C
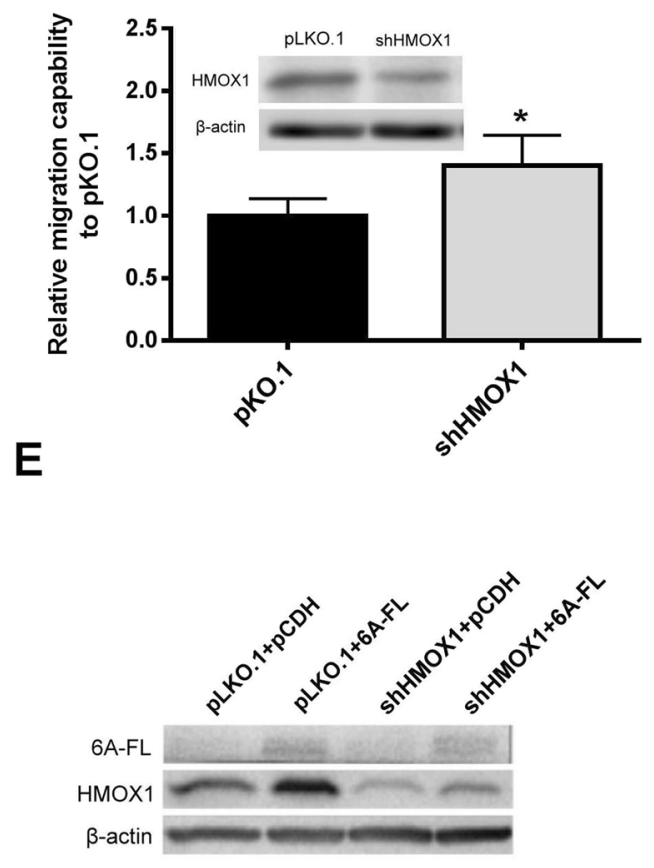

B

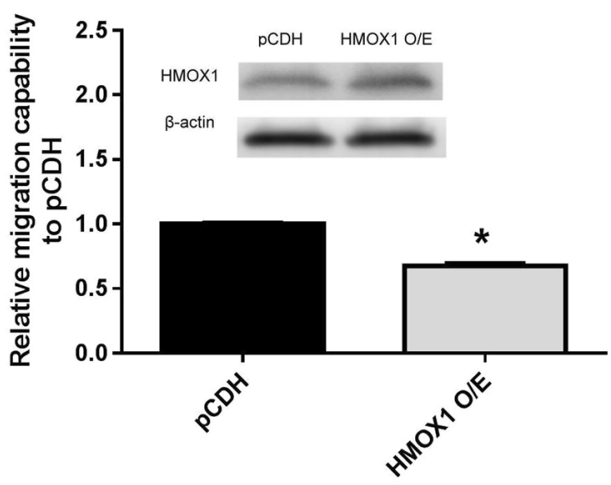

D
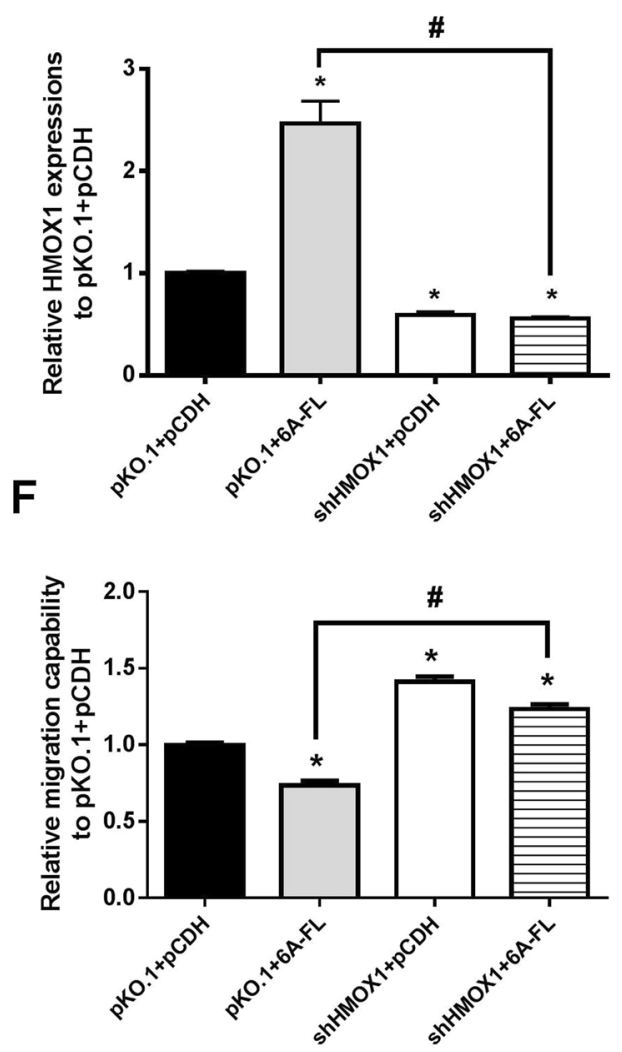

Figure 3. Expression of HMOX1 and migration capability by transwell assay of H1299 cells transfected with different vectors. (A) Relative mRNA expressions of HMOX1 in HMOX1 -overexpressing (HMOX1 O/E) (left) or -silenced (shHMOX1) (right) H1299 cells. (B) Migration capability of H1299 cells overexpressing HMOX1 or empty vector $(\mathrm{pCDH})$. Inset: western blot of HMOX1. (C) Migration capability of HMOX1-silenced H1299 cells and cells containing empty vector (pLKO.1). Inset: western blot of HMOX1. (D) mRNA and (E) protein expression of HMOX1 in H1299 cells co-transfected with either pLKO.1 + pCDH, pLKO.1 + 6A-FL, shHMOX1 + pCDH, or shHMOX1 +6A-FL. (F) Migration capability of H1299 cells co-transfected with either pLKO.1 + pCDH, pLKO.1+6A-FL, shHMOX1 + pCDH, or shHMOX1 + 6A-FL. *Statistical significance compared to empty vector-transfected $\mathrm{H} 1299$ cells at $p<0.05, \mathrm{n}=3$. \# Statistical significance compared to each other at $p<0.05, \mathrm{n}=3$.

Nuclear Factor, Erythroid 2 Like 2 (NRF2) knockdown reverses the effect of SEMA6A on HMOX1 and migration in H1299 cells. NRF2 was reported as an upstream regulator of HMOX1 in another lung cancer cell line, $\mathrm{H} 292^{20}$. Although NRF2 was not included in the first selection, the results of microarray showed that NRF2 was 1.3x up-regulated in SEMA6A-overexpressing H1299 cells. Moreover, 6A-FL-overexpressing H1299 cells had a significantly higher expression of NRF2 determined by RT-qPCR compared to control cells (Fig. 5A). Therefore, we also investigated if SEMA6A uses NRF2 to induce HMOX1, followed by inhibition of migration in H1299 cells. Similar to HMOX1 knockdown, NRF2 knockdown decreased expression of HMOX1 and increased the migration rate of H1299 cells (Fig. 5A,B, open bars). Moreover, it significantly lowered the 
A

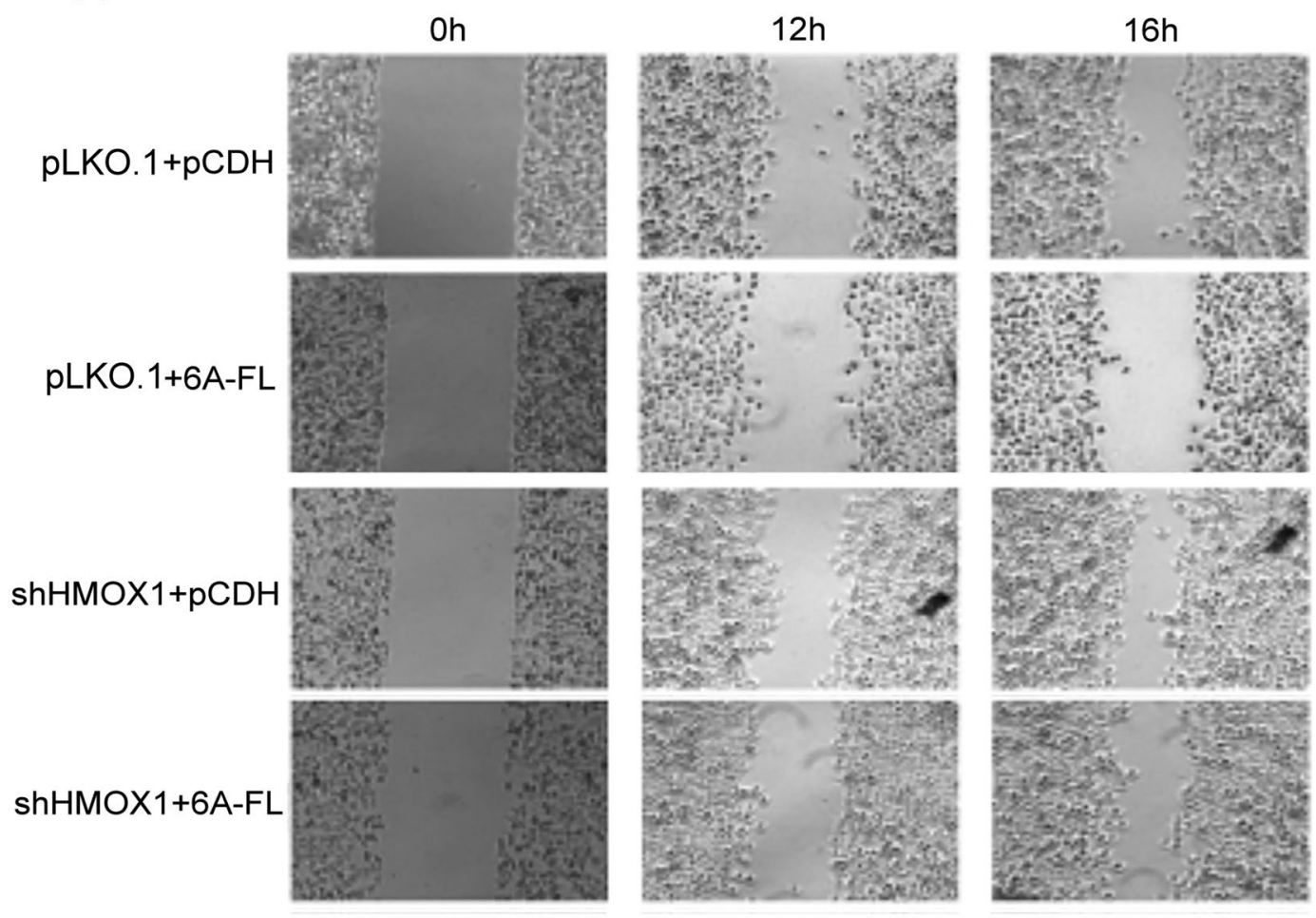

B

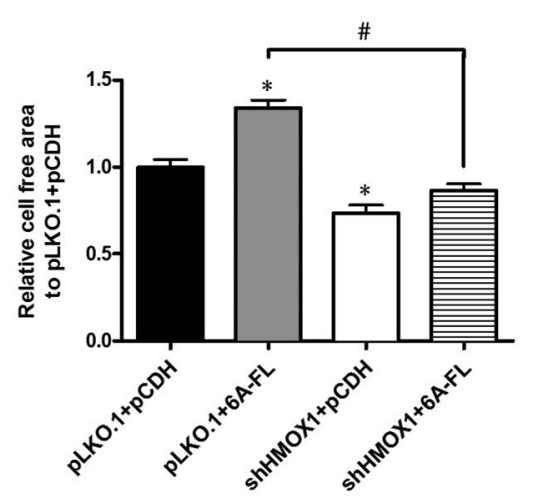

C

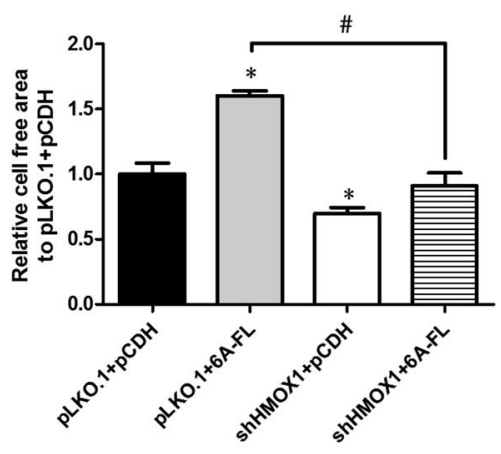

Figure 4. Migration capability evaluated by wound-healing assays. (A) Representative wound healing pictures at $0 \mathrm{~h}, 12 \mathrm{~h}$, and $16 \mathrm{~h} .(\mathbf{B}, \mathbf{C})$ Mobility rate histograms of each group at $12 \mathrm{~h}(\mathbf{B})$ and $16 \mathrm{~h}(\mathbf{C})$, expressed as the cell-free area. *Statistical significance compared to empty vectors (pLKO.1 + pCDH) at $p<0.05$. "Statistical significance compared to each other at $p<0.05$.

expression of HMOX1 in 6A-FL-overexpressing H1299 cells, which was associated with an increase in migration rate (Fig. 5A,B, striped bars). These results indicate that NRF2 is an important upstream mediator of the action of HMOX1 in SEMA6A-induced attenuation of migration.

SEMA6A regulates mRNA expression of Plasminogen Activator, Urokinase (PLAU), Insulin Like Growth Factor Binding Protein 3 (IGFBP3), Matrix metalloproteinase (MMP)-1, and MMP9 via HMOX1. For improving the understanding of SEMA6A-derived migration pathway, we also determined the mRNA expression levels of PLAU, MMP1 and MMP9 using RT-qPCR, because these genes were indicated to be reduced by HMOX1 and to induce migration in cancer cells ${ }^{20-22}$. Moreover, IGFBP3 was up-regulated by HMOX1, which attenuated migration capability of cancer cells ${ }^{22}$. The results showed that overexpression of 6A-FL decreased the mRNA levels of PLAU, MMP1, and MMP9 and increased the mRNA levels of IGFBP3 (Fig. 6). The 6A-FL-induced alterations were reversed when HMOX1 was silenced in 6A-FL-overexpressing H1299 cells. 
A

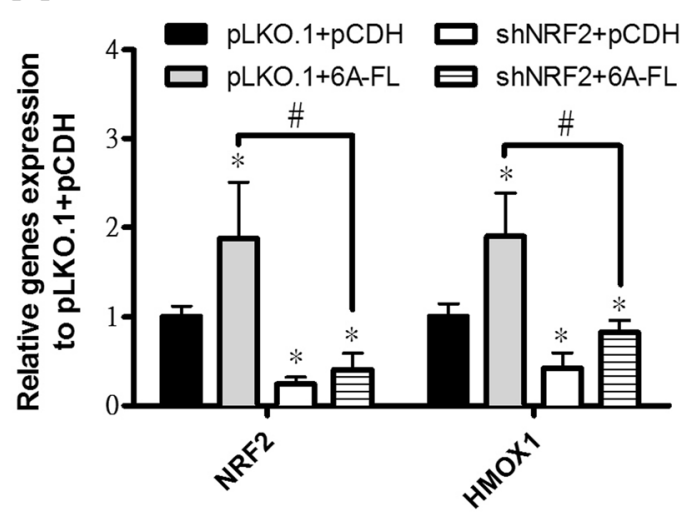

B

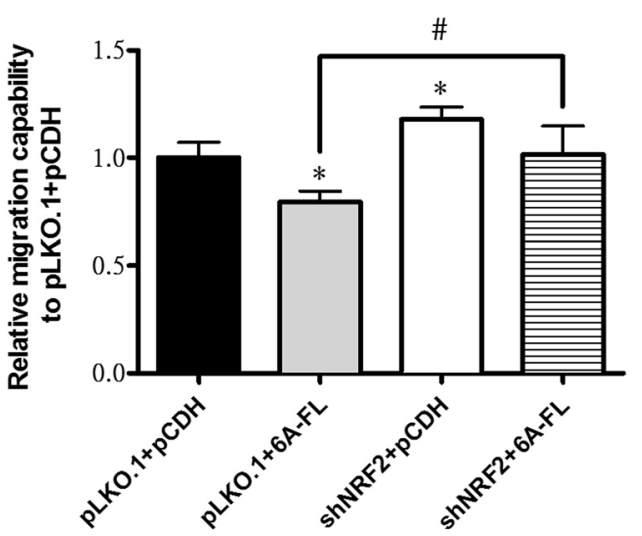

Figure 5. Effect of NRF2 on gene expression and migration capability of H1299 cells. (A) mRNA expression of NRF2 and HMOX1 and (B) migration capability in H1299 cells co-transfected with either pLKO.1 + pCDH, pLKO.1 + 6A-FL, shNRF2 + pCDH, or shNRF2 +6A-FL. *Statistical significance compared to empty vectors $(\mathrm{pLKO} .1+\mathrm{pCDH})$ at $p<0.05$. ${ }^{*}$ Statistical significance compared to each other at $p<0.05$.

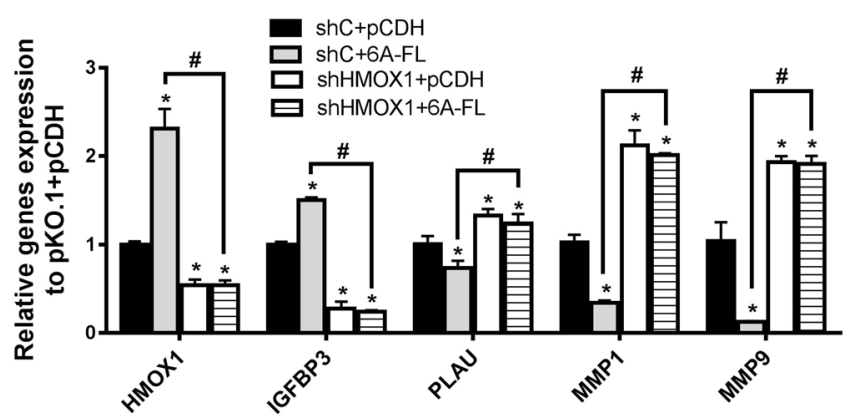

Figure 6. mRNA expression of IGFBP3, PLAU, MMP1, and MMP9 in H1299 cells. Cells were co-transfected with either pLKO.1 + pCDH, pLKO.1 + 6A-FL, shHMOX1 + pCDH, or shHMOX1 + 6A-FL. *Statistical significance compared to empty vectors ( $\mathrm{pLKO} .1+\mathrm{pCDH})$ at $p<0.05$. ${ }^{\text {SStatistical significance compared to }}$ each other at $p<0.05$.

\section{Discussion}

Our data highlight a novel feature of SEMA6A, which is that SEMA6A can reduce lung cancer cell migration through the NRF2/HMOX1 axis in a manner dependent on its cytosolic region. The SEMA6A-derived regulation pathway in migration was also found to occur in other lung cancer cell lines, such as A549 and CL1-5 (Supplementary Fig. 1). Moreover, we demonstrated that SEMA6A employs HMOX1 to regulate the expression of genes related to cell migration, such as PLAU, MMP1, MMP9, and IGFBP3. This newly discovered function and mechanism of SEMA6A indicates that SEMA6A may be a cancer suppressor and can be a biomarker of lung cancer.

Increased cell migration is a characteristic of cancer recurrence and results in low efficacy of cancer treatment $^{2,3}$. Since low levels of SEMA6A are accompanied by high recurrence of cancer ${ }^{19}$, we hypothesized that SEMA6A plays a role in cancer cell migration. SEMA6A was initially described as a regulator of migration in neurons, granule cells, and endothelial cells ${ }^{10,23-25}$. Although overexpression of the extracellular region of SEMA6A in cancer cells suppressed the migration of vascular endothelial cells, the effect of SEMA6A on the migration of cancer cells themselves is still unknown ${ }^{10}$. In the present study, we demonstrated that SEMA6A plays a role in suppressing migration in H1299 cells, which had a lower migration rate when overexpressing 6A-FL, but a higher migration rate when SEMA6A was silenced. Since some semaphorins, such as SEMA6D and SEMA1A, have been reported to function as both receptors and ligands, we further investigated the possibility of SEMA6A reducing cell migration via reverse or forward signaling. Reverse but not forward signaling involves a membrane protein transducing intracellular signals through its cytoplasmic domain(s), and the fact that the SEMA6A's ectodomain construct had no effect on migration capability suggests that SEMA6A-derived migration suppression may be not through forward signaling but reverse signaling, due to its dependence on its cytosolic region.

Our results also demonstrated that SEMA6A modulated migration by inducing the NRF2/HMOX1 axis in H1299 cells. In most types of lung tumors, high intratumoral levels of NRF2 and HMOX1 are accompanied by poor clinical outcomes ${ }^{26,27}$. Moreover, Tertil et al. (2015) reported that inducing the NRF2/HMOX1 axis 
could significantly decrease the migration capability of lung cancer cells ${ }^{20}$. NRF2 has been considered a tumor suppressor because Nrf2-deficient mice are more susceptible to carcinogens ${ }^{28,29}$, and the loss of $N r f 2$ enhances the migration of cancer cells ${ }^{20,30,31}$. NRF2 is regulated by several genes and pathways at the transcriptional and post-transcriptional levels ${ }^{32}$. In light of the observed increase of NRF2 mRNA upon overexpression of 6A-FL, SEMA6A probably regulates NRF2 at the transcriptional level. The aryl hydrocarbon receptor (AhR) and aryl hydrocarbon receptor nuclear translocator (ARNT) complex is one of the transcription factors of NRF2, and the activity of this complex has been suggested to reduce cancer metastasis through increasing the expression of NRF2 $2^{32-34}$. Therefore, SEMA6A might induce NRF2 by regulating either activation or expression of AhR and ARNT in H1299 cells.

HMOX1 was reported as a downstream effector of NRF2 in the migration suppression pathway in lung cancer cells ${ }^{20}$. Traditionally, HMOX1 is known as an enzyme that maintains cellular homeostasis under stress ${ }^{35}$. However, there are more and more studies revealing the functional role of HMOX1 in cancer. For example, overexpression of HMOX1 in lung cancer cells attenuated the expression of matrix metalloproteinases (MMPs), which enhance the migration of cancer cells ${ }^{20,36}$. In the present study, we also demonstrated that HMOX1 was employed by SEMA6A to reduce MMP1 and MMP9 (Fig. 6). In addition, HMOX1 up-regulated E-cadherin and $\beta$-catenin expression, increased filopodia zippering at the leading edge of cells, and favored a more epithelial phenotype ${ }^{37,38}$. Recently, RNA-seq data from prostate cancer cells overexpressing HMOX1 showed that HMOX1 down-modulated the PLAU pathway related to cell adhesion and cell-cell communication. They presented that HMOX1 reduced PLAU directly affecting Rho GTPases through the alpha V-Beta 3 integrin receptor, which in turn increased the potentiality of tumor cells to adhere to each other ${ }^{38}$. Moreover, to silence PLAU led to a reduced capability of migration and invasion in lung cancer cell lines, H1299 and A54921. Since the expression of PLAU was regulated by HMOX1 in 6A-FL-overexpressing H1299, PLAU might also be involved in the SEMA6A-induced pathway of migration suppression.

IGFBP3 was another gene induced in 6A-FL-overexpressing cells. Overexpression of IGFBP3 reduced migration and invasion by down-regulating PLAU in lung cancer cell lines H1299 and A54922. Torng et al. (2008) showed that IGFBP3 was one of the significantly suppressed genes in endometrioid carcinoma cells with high invasion $^{39}$. Moreover, low expression of IGFBP3 correlated clinically with higher tumor grade, advanced stage, and poor survival ${ }^{39,40}$. In our study, 6A-FL overexpression induced IGFBP3, while the induction of IGFBP3 was reversed if HMOX1 was silenced in the 6A-FL-overexpressing cells. Therefore, IGFBP3 is another potential candidate involved in the SEMA6A-induced migration suppression pathway.

In conclusion, the present study identified a novel suppressor of lung cancer cell migration, SEMA6A, which attenuates migration by inducing the NRF2/HMOX1 axis. Our results not only explain why low SEMA6A expression correlates with high recurrence rates in clinical trials, but also suggest that SEMA6A could be exploited in cancer therapy.

\section{Materials and Methods}

Cell culture. The HEK-293T cell line was provided by Dr. Shau-Ping Lin (Institute of Biotechnology, National Taiwan University) and cultured in DMEM (GIBCO, CA, USA), and the H1299 cell line was purchased from Bioresource Collection and Research Center (Hsinchu, Taiwan) and cultured in RPMI-1640 (GIBCO, CA, USA). All cells were incubated with medium containing $10 \%$ fetal bovine serum (FBS) and $1 \%$ penicillin/streptomycin at $37^{\circ} \mathrm{C}$ in a $5 \% \mathrm{CO}_{2}$ humidified incubator.

RNA extraction and reverse transcription. Total RNA was extracted from cells using an RNeasy Mini kit (Qiagen, Hilden, Germany), and $1 \mu \mathrm{g}$ of RNA from each sample was reverse-transcribed using a High-Capacity cDNA Reverse Transcription kit (Applied Biosystems, Foster City, CA, USA), according to the instructions of the manufacturer.

Gene constructs and shRNA. 6A-FL and HMOX1 were generated from Hs68 and H1299 cells, respectively, using RT-PCR. 6Aect was produced from the 6A-FL sequence. 6A-FL and 6Aect were given a $6 \mathrm{xHis}$ tag sequence at the $\mathrm{C}$-terminus. The primers for these constructs are listed in Supplementary Table 1. The shRNAs for SEMA6A (TRCN0000061108 and TRCN0000061109), HMOX1 (TRCN0000290436), and NRF2 (TRCN0000007558) were purchased from the RNAi Core Facility (Academia Sinica, Taipei, Taiwan).

Virus production and cell infection. HEK-293T cells $\left(4 \times 10^{6}\right.$ cells $)$ were co-transfected with packaging plasmid (psPAX2; $6 \mu \mathrm{g}$ ), envelope plasmid (pMD2G; $2 \mu \mathrm{g}$ ), and the transfer vector plasmid (pCDH-CMV-MCS-EF1-puro for overexpression; pLKO.1 for gene silence; $8 \mu \mathrm{g}$ ), which was empty or contained either target genes or shRNA, using TransIT ${ }^{\circledR}-2020$ Transfection Reagent (Mirus Bio LLC). The supernatants were collected at 48 and $72 \mathrm{~h}$ post-transfection and stored at $-80^{\circ} \mathrm{C}$. H1299 cells were seeded in 24 -well plates at $4 \times 10^{4}$ cells/well and incubated for $24 \mathrm{~h}$, and then $1 \mathrm{~mL}$ of viral supernatant was added into each well and centrifuged at $2500 \mathrm{rpm}$ for $60 \mathrm{~min}$ at room temperature followed by incubation at $37^{\circ} \mathrm{C}$ in $5 \% \mathrm{CO}_{2}$.

Western blotting. For protein extraction, cells were lysed by RIPA lysis buffer (Millipore, CA, USA) containing protease inhibitor cocktail, $10 \mathrm{mM} \beta$-glycerophosphate, and $5 \mathrm{mM} \mathrm{Na}_{3} \mathrm{VO}_{4}$ followed by sonicating homogenization for $20 \mathrm{~s}$ on ice. The protein concentration was measured by the Bradford protein assay (Bio-Rad, Hercules, CA, USA). Equal amounts of protein from each sample were separated by $10 \%$ SDS-PAGE and then transferred onto a nitrocellulose membrane (Millipore). After incubation overnight at $4{ }^{\circ} \mathrm{C}$ with primary antibodies against $6 \times$ His (Millipore), SEMA6A (Genetex, Hsinchu,Taiwan), HMOX1 (Millipore), or $\beta$-actin (Millipore), the samples were hybridized with horseradish peroxidase (HRP)-conjugated secondary antibodies at room temperature for $1 \mathrm{~h}$. Finally, HRP activity was visualized by an enhanced chemiluminescence system (UVP BioSpectrum Imaging System). 
Transwell assay. The $6.5 \mathrm{~mm}$-diameter cell culture inserts $(8 \mu \mathrm{m}$ pore size $)$ were used to perform transwell assays in 24 -well plates. After starving the cells with serum-free medium for $24 \mathrm{~h}$, they were seeded at $5 \times 10^{4}$ cells/well in the upper chamber of the filter in $200 \mu \mathrm{L}$ of FBS-free medium. Then, $0.75 \mathrm{~mL}$ of complete medium containing $10 \%$ FBS was added in the lower chamber. After $16 \mathrm{~h}$, cells were fixed for $15 \mathrm{~min}$ at room temperature with $10 \%$ acetic acid containing $10 \%$ methanol, and washed with $1 \times$ PBS. The non-migrating cells on the inner transwell membrane were wiped carefully, and the membranes were stained with $1 \mathrm{~mL}$ of $0.5 \%$ crystal violet for $30 \mathrm{~min}$ and washed with water. The stained cells were solubilized with $10 \%$ acetic acid and quantitated on a microplate reader at $580 \mathrm{~nm}$.

Microarray data analysis. Microarray data were from our previous unpublished studies, and the intensity data of SEMA6A were analyzed using Partek software (Partek, Chesterfield, MO, USA) to obtain mRNA expression levels. Robust multi-array average analysis was used to preprocess probe-level expression data of SEMA6A, including background correction, quantile normalization, and summarization. The genes were selected when their fold changes were $>1.5$ and $\mathrm{p}$ values were $<0.01$ using paired $\mathrm{t}$-tests. Functional grouping and pathway analysis were performed using IPA software (Ingenuity Systems, Redwood City, CA, USA).

Quantitative PCR. Quantitative PCR was processed using an ABI 7300 instrument (Applied Biosystems, Foster City, CA, USA) with a SYBR Green Master Mix kit (Applied Biosystems) according to standard procedures. The sequences of the gene-specific primers (Purigo, Taipei, Taiwan) are listed in Supplementary Table 1. GAPDH was used as an internal control for normalizing the mRNA levels of tested genes.

Wound-healing assay. Approximately equal amount of cells were loaded into the left and right chambers of an ibidi Culture-Insert (Applied BioPhysics, NY, NY, USA). Each insert was placed in one well of a 24-well plate. The experiments were carried out in triplicate. After overnight incubation at $37^{\circ} \mathrm{C}$ and $5 \% \mathrm{CO}_{2}$, the insert was removed and a photo of each well was taken at 0,12 , and $16 \mathrm{~h}$. The cell-free area was measured by Image software at every time point.

Statistical analysis. A two-tailed Student's t-test was applied to all the data in this study. Differences were considered to be significant if $p<0.05$. All values in the study are presented as mean \pm standard error of mean of three or more experiments.

\section{References}

1. Hanahan, D. \& Weinberg, R. A. The hallmarks of cancer. Cell 100, 57-70 (2000).

2. Mehlen, P. \& Puisieux, A. Metastasis: a question of life or death. Nat Rev Cancer 6, 449-458 (2006).

3. Chanvorachote, P. \& Chunhacha, P. Lung Cancer Metastasis. Introduction to Cancer Metastasis, 61-76 (2016)

4. Siegel, R. L., Miller, K. D. \& Jemal, A. Cancer Statistics, 2017. Ca-Cancer J. Clin. 67, 7-30, https://doi.org/10.3322/caac.21387 (2017).

5. Chakraborty, G., Kumar, S., Mishra, R., Patil, T. V. \& Kundu, G. C. Semaphorin 3A suppresses tumor growth and metastasis in mice melanoma model. Plos One 7, e33633, https://doi.org/10.1371/journal.pone.0033633 (2012).

6. Shahi, P. et al. GATA3 targets semaphorin 3B in mammary epithelial cells to suppress breast cancer progression and metastasis. Oncogene 36, 5567-5575 (2017).

7. Gao, X. S. et al. Semaphorin-3F functions as a tumor suppressor in colorectal cancer due to regulation by DNA methylation. Int. J. Clin. Exp. Patho. 8, 12766-12774 (2015).

8. Shen, C. Y. et al. The extracellular SEMA domain attenuates intracellular apoptotic signaling of semaphorin $6 \mathrm{~A}$ in lung cancer cells. Oncogenesis 7 (2018)

9. Lu, T. P. et al. Identification of a Novel Biomarker, SEMA5A, for Non-Small Cell Lung Carcinoma in Nonsmoking Women. Cancer Epidem. Biomar. 19, 2590-2597 (2010).

10. Dhanabal, M. et al. Recombinant Semaphorin 6A-1 ectodomain inhibits in vivo growth factor and tumor cell line-induced angiogenesis. Cancer Biol Ther 4, 659-668 (2005).

11. Tran, T. S., Kolodkin, A. L. \& Bharadwaj, R. Semaphorin regulation of cellular morphology. Annu Rev Cell Dev Biol 23, 263-292, https://doi.org/10.1146/annurev.cellbio.22.010605.093554 (2007).

12. Yaron, A., Huang, P. H., Cheng, H. J. \& Tessier-Lavigne, M. Differential requirement for Plexin-A3 and -A4 in mediating responses of sensory and sympathetic neurons to distinct class 3 Semaphorins. Neuron 45, 513-523, https://doi.org/10.1016/j. neuron.2005.01.013 (2005).

13. Winberg, M. L. et al. Plexin A is a neuronal semaphorin receptor that controls axon guidance. Cell 95, 903-916 (1998).

14. Li, X. \& Lee, A. Y. Semaphorin 5A and plexin-B3 inhibit human glioma cell motility through RhoGDIalpha-mediated inactivation of Racl GTPase. J Biol Chem 285, 32436-32445, https://doi.org/10.1074/jbc.M110.120451 (2010).

15. Xu, X. M. et al. The transmembrane protein semaphorin 6A repels embryonic sympathetic axons. J Neurosci 20, 2638-2648 (2000).

16. Runker, A. E. et al. Mutation of Semaphorin-6A disrupts limbic and cortical connectivity and models neurodevelopmental psychopathology. Plos One 6, e26488, https://doi.org/10.1371/journal.pone.0026488 (2011).

17. Runker, A. E., Little, G. E., Suto, F., Fujisawa, H. \& Mitchell, K. J. Semaphorin-6A controls guidance of corticospinal tract axons at multiple choice points. Neural Dev 3, 34, https://doi.org/10.1186/1749-8104-3-34 (2008).

18. Klostermann, A., Lutz, B., Gertler, F. \& Behl, C. The orthologous human and murine semaphorin 6A-1 proteins (SEMA6A-1/ Sema6A-1) bind to the enabled/vasodilator-stimulated phosphoprotein-like protein (EVL) via a novel carboxyl-terminal zyxin-like domain. J Biol Chem 275, 39647-39653, https://doi.org/10.1074/jbc.M006316200 (2000).

19. Chien, M. N. et al. Recurrence-associated genes in papillary thyroid cancer: An analysis of data from The Cancer Genome Atlas. Surgery 161, 1642-1650, https://doi.org/10.1016/j.surg.2016.12.039 (2017).

20. Tertil, M. et al. Nrf2-heme oxygenase-1 axis in mucoepidermoid carcinoma of the lung: Antitumoral effects associated with downregulation of matrix metalloproteinases. Free Radic Biol Med 89, 147-157, https://doi.org/10.1016/j.freeradbiomed.2015.08.004 (2015).

21. Lakka, S. S. et al. Adenovirus-mediated antisense urokinase-type plasminogen activator receptor gene transfer reduces tumor cell invasion and metastasis in non-small cell lung cancer cell lines. Clin Cancer Res 7, 1087-1093 (2001).

22. Oh, S. H. et al. Antimetastatic activity of insulin-like growth factor binding protein-3 in lung cancer is mediated by insulin-like growth factor-independent urokinase-type plasminogen activator inhibition. Mol Cancer Ther 5, 2685-2695, https://doi. org/10.1158/1535-7163.MCT-06-0142 (2006).

23. Renaud, J. et al. Plexin-A2 and its ligand, Sema6A, control nucleus-centrosome coupling in migrating granule cells. Nat. Neurosci. 11, 440-449 (2008) 
24. Bron, R. et al. Boundary cap cells constrain spinal motor neuron somal migration at motor exit points by a semaphorin-plexin mechanism. Neural Dev. 2 (2007).

25. Segarra, M. et al. Semaphorin 6A regulates angiogenesis by modulating VEGF signaling. Blood 120, 4104-4115 (2012).

26. Solis, L. M. et al. Nrf2 and Keap1 Abnormalities in Non-Small Cell Lung Carcinoma and Association with Clinicopathologic Features. Clin. Cancer Res. 16, 3743-3753 (2010).

27. Tsai, J. R. et al. High expression of heme oxygenase-1 is associated with tumor invasiveness and poor clinical outcome in non-small cell lung cancer patients. Cell Oncol. 35, 461-471 (2012).

28. Ramos-Gomez, M., Dolan, P. M., Itoh, K., Yamamoto, M. \& Kensler, T. W. Interactive effects of nrf2 genotype and oltipraz on benzo[a]pyrene-DNA adducts and tumor yield in mice. Carcinogenesis 24, 461-467 (2003).

29. Iida, K. et al. Nrf2 is essential for the chemopreventive efficacy of oltipraz against urinary bladder carcinogenesis. Cancer Res. 64, 6424-6431 (2004).

30. Satoh, H. et al. Nrf2-deficiency creates a responsive microenvironment for metastasis to the lung. Carcinogenesis 31, 1833-1843 (2010).

31. Rachakonda, G. et al. Increased cell migration and plasticity in Nrf2-deficient cancer cell lines. Oncogene 29, 3703-3714 (2010)

32. Hayes, J. D. \& Dinkova-Kostova, A. T. The Nrf2 regulatory network provides an interface between redox and intermediary metabolism. Trends Biochem. Sci. 39, 199-218 (2014).

33. Huang, C. R. et al. Down-regulation of ARNT promotes cancer metastasis by activating the fibronectin/integrin beta 1/FAK axis. Oncotarget 6, 11530-11546 (2015).

34. Tsai, C. H. et al. The inhibition of lung cancer cell migration by AhR-regulated autophagy. Sci. Rep. 7, 41927 (2017).

35. Dulak, J. \& Jozkowicz, A. Novel Faces of Heme Oxygenase-1: Mechanisms and Therapeutic Potentials. Antioxid. Redox Signal. 20, 1673-1676 (2014).

36. Gialeli, C., Theocharis, A. D. \& Karamanos, N. K. Roles of matrix metalloproteinases in cancer progression and their pharmacological targeting. Febs J. 278, 16-27 (2011).

37. Gueron, G. et al. Heme-oxygenase-1 implications in cell morphology and the adhesive behavior of prostate cancer cells. Oncotarget 5, 4087-4102 (2014).

38. Paez, A., Vazquez, E. \& Gueron, G. Heme oxygenase 1 governs the cytoskeleton at filopodia: pulling the brakes on the migratory capacity of prostate tumoral cells. Cell Death Discov 3, 17020, https://doi.org/10.1038/cddiscovery.2017.20 (2017).

39. Torng, P. L. et al. Insulin-like growth factor binding protein-3 (IGFBP-3) acts as an invasion-metastasis suppressor in ovarian endometrioid carcinoma. Oncogene 27, 2137-2147, https://doi.org/10.1038/sj.onc.1210864 (2008).

40. Yu, H. \& Rohan, T. Role of the insulin-like growth factor family in cancer development and progression. J Natl Cancer Inst $\mathbf{9 2}$, 1472-1489 (2000).

\section{Acknowledgements}

This work was supported by Ministry of Science and Technology, Taiwan (106-2314-B-002 -081 -MY3). We thank the National RNAi Core Facility at Academia Sinica in Taiwan for providing shRNA reagents and related services, and Melissa Stauffer, Ph.D., for editing the manuscript.

\section{Author Contributions}

L.H.C. and C.Y.L. designed and performed the experiments and wrote the paper; L.C.L. wrote the paper; M.H.T. and E.Y.C. designed the experiments, supervised the project, and wrote the paper. All authors discussed the results and contributed to the manuscript.

\section{Additional Information}

Supplementary information accompanies this paper at https://doi.org/10.1038/s41598-019-49874-8.

Competing Interests: The authors declare no competing interests.

Publisher's note Springer Nature remains neutral with regard to jurisdictional claims in published maps and institutional affiliations.

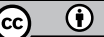

Open Access This article is licensed under a Creative Commons Attribution 4.0 International License, which permits use, sharing, adaptation, distribution and reproduction in any medium or format, as long as you give appropriate credit to the original author(s) and the source, provide a link to the Creative Commons license, and indicate if changes were made. The images or other third party material in this article are included in the article's Creative Commons license, unless indicated otherwise in a credit line to the material. If material is not included in the article's Creative Commons license and your intended use is not permitted by statutory regulation or exceeds the permitted use, you will need to obtain permission directly from the copyright holder. To view a copy of this license, visit http://creativecommons.org/licenses/by/4.0/.

(C) The Author(s) 2019 